\title{
Additional Mass: Orthotropic Membrane Material with Four Sides Fixed in Air Flow
}

\author{
Zhiying Tian1, Weiju Song2*, Yafei Zhang², Huimin Yin², Xinxin Wang2 \\ ${ }^{1}$ College of Civil Engineering, City College of Science and Technology, Chongqing University, Chongqing, China \\ ${ }^{2}$ College of Civil Engineering, Hebei University of Engineering, Handan, China \\ Email: *nimrodsong@126.com
}

How to cite this paper: Tian, Z.Y., Song, W.J., Zhang, Y.F., Yin, H.M. and Wang, X.X. (2020) Additional Mass: Orthotropic Membrane Material with Four Sides Fixed in Air Flow. Journal of Applied Mathematics and Physics, 8, 956-967.

https://doi.org/10.4236/jamp.2020.86074

Received: May 1, 2020

Accepted: May 28, 2020

Published: June 1, 2020

Copyright $\odot 2020$ by author(s) and Scientific Research Publishing Inc. This work is licensed under the Creative Commons Attribution International License (CC BY 4.0).

http://creativecommons.org/licenses/by/4.0/

\section{Open Access}

\begin{abstract}
When the membrane material in the air field vibrates, it will drive the movement of the surrounding air. The aerodynamic force generated by the moving air will act on the membrane material in turn, resulting in the change of dynamic characteristics such as membrane vibration frequency. In this paper, the additional air mass produced by membrane vibration in air is studied. Firstly, under the assumption that the incoming flow is uniform and incompressible ideal potential flow, the additional air mass acting on the surface is derived by using the thin airfoil theory and potential flow theory respectively. Then, according to the first law of thermodynamics and the principle of aeroelasticity, the analytical expression of the additional air mass is derived. Finally, through a specific example, the variation of the additional air mass with the membrane material parameters and pretension, as well as the influence of the aerodynamic force on the vibration frequency and amplitude of the membrane is obtained.
\end{abstract}

\section{Keywords}

Membrane Material, Additional Mass, Incompressible Fluid Motion, Wind Speed

\section{Introduction}

In the process of its vibration, the membrane material in the air fluid will cause the movement of air in a certain range around. Obviously, the acceleration or deceleration of the membrane material in the process of vibration will drive the acceleration or deceleration of the surrounding air. However, the accelerating or decelerating motion of the surrounding air will act on the vibrating membrane, so it will cause the change of its vibration frequency and other dynamic charac- 
teristics. It is inevitable that the mass of the membrane should include the added value brought by the movement of the surrounding air in addition to its own properties. For most traditional structures, such as steel structure and concrete structure, the value of additional mass is very small compared with the structure itself, so it can be ignored in the analysis of structural response. But unfortunately, for the structure of membrane material, the additional mass is equal to or even more than the mass of membrane itself. Obviously, for the study of nonlinear vibration of membrane, the additional mass becomes a more important factor.

Miyake (1992) studied the added mass of the flexible plate in the stable fluid by analytical method. The results show that the added mass decreases with the increase of the number of vibration modes of the plate [1]; Li (2011), Wang (2011), Zhou (2014) studied the additional mass of films with different shapes under different air densities through theoretical calculation, numerical analysis and a series of tests, and gave the additional mass coefficient of about 0.65 [2] [3] [4]; Kim (2011), and the research of scholars such as Wu (2003) and Yang (2008, 2010) proposed a calculation method to simulate the wind structure interaction effect of tension structure. The added mass and aerodynamic damping are measured by experiments. Furthermore, the change of interaction parameters and its influence on free vibration characteristics are studied. And the mechanism of wind structure interaction is discussed. The results show that the ratio of added mass to self mass is about 0.2 to 1.2 [5] [6] [7]. Sun deduced the analytical expressions of additional mass and aerodynamic damping based on the principle of energy conservation, and analyzed the dynamic effects of open membrane structure and closed membrane structure respectively [8]. Li deduced the analytical expressions of additional mass, radiation damping and air bearing stiffness, and analyzed the influence parameters systematically [9].

In this paper, the additional mass produced by the vibration of membrane materials in the air is studied by analytical method with the consideration of the orthotropic characteristics of the membrane. Firstly, assuming that the incoming flow is a uniform incompressible ideal potential flow, the aerodynamic forces acting on the surface are derived by using the thin airfoil theory and the potential flow theory [10] [11]. Then, using the first law of thermodynamics and the principle of aeroelasticity, the analytical expression of additional mass is derived. Finally, the variation of the additional mass with the membrane material parameters and pretension is obtained by a specific example. The research results provide reasonable design reference for engineers, enrich the design theory of membrane structure, and further ensure the structural safety of tension membrane structure under wind load.

\section{Derivation of Aerodynamic Force of Membrane Materials}

The membrane material with four sides fixed in the air flow field is shown in Figure 1. 
For the membrane material of air, due to the small membrane thickness, air flows from both sides of the membrane surface, which can be approximately determined by the thin wing theory. In the analysis, the vortex surface is used instead of the membrane surface, as shown in Figure 2. The vortex distribution per unit area of the membrane is defined as $\gamma_{c}(x, y, t)$; the wake vortex force generated by the trailing edge of the membrane per unit time $(x>A)$ is defined as $\gamma_{w}(x, y, t)$. Within the span of the membrane, the force generated by the surface vortex per unit length is represented by the vortex intensity (ring density), as shown in Figure 3.

According to thin wing theory [1],

$$
\gamma_{c}=\lim _{\Delta S \rightarrow 0}\left(\frac{\int \boldsymbol{V} \mathrm{d} \boldsymbol{S}}{\Delta S}\right)
$$

where $\Delta S$ is the width of the vortex surface enclosed by the circumference.

Suppose that $p_{1}$ is the indoor air pressure of the lower surface of the membrane, $p_{2}$ is the outdoor air pressure of the upper surface of the membrane, and that the incoming flow with the velocity $V$ moves in the $X$ direction without rotation. Then, according to the Bernoulli equation, the following equation can be obtained [12]:

$$
\rho_{0}\left[\frac{\partial \phi_{1}}{\partial t}+\frac{1}{2}\left(v_{x 1}^{2}+v_{y 1}^{2}+v_{z 1}^{2}\right)\right]+p_{1}=\rho_{0}\left[\frac{\partial \phi_{2}}{\partial t}+\frac{1}{2}\left(v_{2}^{x 2}+v_{2}^{y 2}+v_{2}^{z 2}\right)\right]+p_{2}
$$

Figure 1. Schematic diagram of membrane material.

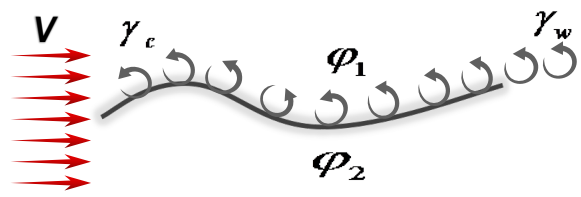

Figure 2. Schematic plan of vortex sheet.

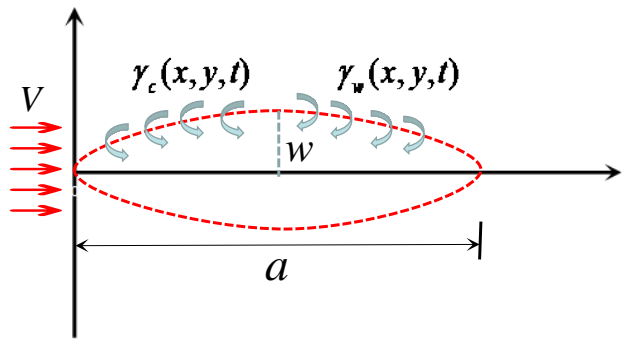

Figure 3. Schematic diagram of vibrating membrane vortex. 
where, $\phi_{i}$ is the velocity potential function of the upper and lower surfaces of the membrane, and $v_{x i}, v_{y i}, v_{z i}$ are the velocity components of the upper and lower surfaces of the membrane respectively. Assuming that the incoming flow is along the $Y$ direction of the structure, the velocity is $V$, and the disturbance velocity in all directions is $\bar{v}_{x i}, \bar{v}_{y i}$ and $\bar{v}_{z i}$ when the flow field encounters obstacles [13], the velocity component in Equation (2) is

$$
v_{x i}=\bar{v}_{x i}, \quad v_{y i}=V+\bar{v}_{y i}, \quad v_{z i}=\bar{v}_{z i}
$$

Generally, $\bar{v}_{x i}, \bar{y}_{y i}, \bar{v}_{z i} \ll V$, then, omitting high order small quantity term has general

$$
v_{x i}^{2}+v_{y i}^{2}+v_{z i}^{2}=\bar{v}_{x i}^{2}+\left(V+\bar{v}_{y i}\right)^{2}+\bar{v}_{z i}^{2} \approx V^{2}+2 \sqrt{y i}
$$

Substituting Equation (3) into Equation (2) and simplifying it, then,

$$
p_{1}-p_{2}=\rho_{0}\left[\left(\frac{\partial \phi_{2}}{\partial t}-\frac{\partial \phi_{1}}{\partial t}\right)+V\left(\bar{v}_{y 2}-\bar{v}_{y 1}\right)\right]
$$

Velocity and velocity potential can be approximately considered as functions of horizontal coordinates $x, y$ and $t$.

$$
\phi^{\prime}=\int_{0}^{x} v_{x} \mathrm{~d} x+\int_{0}^{y} v_{y} \mathrm{~d} y \approx \int_{0}^{x} v_{y} \mathrm{~d} x
$$

Then,

$$
\frac{\partial \phi_{2}}{\partial t}-\frac{\partial \phi_{1}}{\partial t}=\int_{0}^{x}\left(v_{y 2}-v_{y 1}\right) \mathrm{d} x
$$

Assuming that the vortex density on the surface element $\mathrm{d} x \mathrm{~d} y$ is $\gamma_{c}(x, y, t)$, applying the thin airfoil theory, then:

$$
v_{y 2}-v_{y 1}=\gamma_{c}(x, y, t)
$$

Substituting Equations (6) and (7) into Equations (4), the aerodynamic force acting on the membrane unit can be obtained as follows:

$$
p=p_{1}-p_{2}=\rho_{0} \frac{\partial}{\partial t} \int_{0}^{y} \gamma_{c}(x, \eta, t) \mathrm{d} \eta+\rho_{0} V \gamma_{c}
$$

The vortex lattice method is used to solve the expression of $\gamma_{c}(x, y, t)$ in Equation (8). The projection area $\{0 \leq x \leq a, 0 \leq y \leq b\}$ of the membrane on the xoy plane is divided into $M \times N$ vortex grids. The dimensionless vortex strength $\Gamma / a V$ is expressed by $\gamma_{1}, \gamma_{2}, \gamma_{3}, \cdots, \gamma_{M \times N}$. The induced velocity $v_{z i j}$ at the control point $i$ of the jth vortex grid is

$$
\frac{v_{z i j}}{V}=C_{i j} \gamma_{j}
$$

where $C_{i j}$ is the value of $v_{z i j} / V$ generated by $\gamma_{j}$ at point $i$, and the $Z$-induced velocity generated by all horseshoe vortices at point $i$ is

$$
\frac{V_{z i}}{V}=\sum_{j=1}^{M \times N} C_{i j} \gamma_{j}
$$

The horseshoe vortex on the membrane surface is shown in Figure 4. The coordinates of corner points 1 and 2 ar $\left(x_{1 j}, y_{1 j}\right)$ and $\left(x_{2 j}, y_{2 j}\right)$ respectively, and the coordinates of control point $i$ are $\left(x_{i}, y_{i}\right)$, then: 


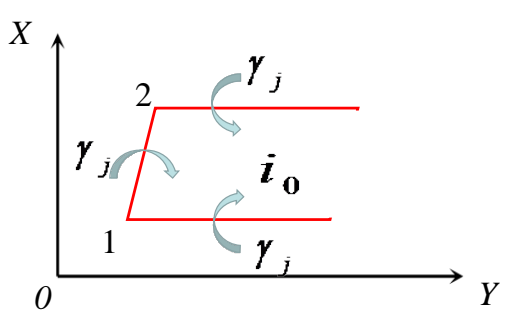

Figure 4. Horseshoe vortex over the membrane surface.

$$
\begin{aligned}
C_{i j}=\left(\frac{v_{z i j}}{V}\right)_{\gamma_{j}=1}= & \frac{a}{4 \pi}\left\{\frac{1}{\left(y_{i}-y_{1 j}\right)\left(x_{i}-x_{2 j}\right)-\left(y_{i}-y_{2 j}\right)\left(x_{i}-x_{1 j}\right)}\right. \\
& \times \frac{\left(y_{2 j}-y_{1 j}\right)\left(y_{i}-y_{1 j}\right)+\left(x_{2 j}-x_{1 j}\right)\left(x_{i}-x_{1 j}\right)}{\sqrt{\left(y_{i}-y_{1 j}\right)^{2}+\left(x_{i}-x_{1 j}\right)^{2}}} \\
& \left.-\frac{\left(y_{2 j}-y_{1 j}\right)\left(y_{i}-y_{2 j}\right)+\left(x_{2 j}-x_{1 j}\right)\left(x_{i}-x_{2 j}\right)}{\sqrt{\left(y_{i}-y_{2 j}\right)^{2}+\left(x_{i}-x_{2 j}\right)^{2}}}\right] \\
& +\frac{1.0}{x_{1 j}-x_{i}}\left[1.0+\frac{y_{i}-y_{1 j}}{\sqrt{\left(y_{i}-y_{1 j}\right)^{2}+\left(x_{i}-x_{1 j}\right)^{2}}}\right] \\
& \left.-\frac{1.0}{x_{2 j}-x_{i}}\left[1.0+\frac{y_{i}-y_{2 j}}{\sqrt{\left(y_{i}-y_{2 j}\right)^{2}+\left(x_{i}-x_{2 j}\right)^{2}}}\right]\right\}
\end{aligned}
$$

The expressions of coordinates of each point in the formula are as follows:

$$
\begin{aligned}
& \left\{\begin{array}{ll}
x_{i}=x_{(l-1) N+k}=\frac{a}{M}\left(l-\frac{1}{2}\right) & (1 \leq l \leq M) \\
y_{i}=y_{(l-1) N+k}=\frac{b}{N}\left(k-\frac{1}{4}\right) & (1 \leq k \leq N)
\end{array}, \quad(i=1,2, \cdots, M \times N)\right. \\
& \left\{\begin{array}{ll}
x_{1 j}=x_{[[(l-1) N+k]}=\frac{a}{M}(l-1) & (1 \leq l \leq M) \\
y_{1 j}=y_{1[(l-1) N+k]}=\frac{b}{N}\left(k-\frac{3}{4}\right) & (1 \leq k \leq N)
\end{array}, \quad(i=1,2, \cdots, M \times N)\right. \\
& \left\{\begin{array}{ll}
x_{2 j}=x_{2[(l-1) N+k]}=\frac{a}{M} l & (1 \leq l \leq M) \\
y_{2 j}=y_{2[(l-1) N+k]}=\frac{b}{N}\left(k-\frac{3}{4}\right) & (1 \leq k \leq N)
\end{array}, \quad(i=1,2, \cdots, M \times N)\right.
\end{aligned}
$$

Applying boundary conditions (11) to the ith control point, then

$$
\frac{V_{z i}}{V}=\sum_{j=1}^{M \times N} C_{i j} \gamma_{j}=\left[\frac{\partial z}{\partial y}+\frac{1}{V} \frac{\partial z}{\partial t}\right]_{i}=\left[\frac{\partial z_{0}}{\partial y}+\frac{\partial w}{\partial y}+\frac{1}{V} \frac{\partial w}{\partial t}\right]_{i} \quad(i=1,2, \cdots, M \times N)
$$

The vibration displacement of the membrane is assumed to be: 


$$
w(x, y, t)=W(x, y) \cdot T(t)
$$

where, $T(t)$ is a function of time in the process of vibration, and $W(x, y)$ is a function of mode shape.

$$
\sum_{j=1}^{M \times N} C_{i j} \gamma_{j}=\frac{\partial W}{\partial y} T(t)+\frac{T^{\prime}(t)}{V} W \quad(i=1,2, \cdots, M \times N)
$$

The value of $\gamma_{j}$ can be obtained by combining the equations on $M \times N$ control points.

Let the expression of $\gamma_{j}$ be

$$
\gamma_{j}=a_{1 j} T(t)+a_{2 j} \frac{T^{\prime}(t)}{V},(j=1,2, \cdots, M \times N)
$$

Substituting Equation (15) into Equation (14), then

$$
\sum_{j=1}^{M \times N} C_{i j}\left(a_{1 j} T(t)+a_{2 j} \frac{T^{\prime}(t)}{V}\right)=\frac{\partial W}{\partial y} T(t)+\frac{T^{\prime}(t)}{V} W
$$

So,

$$
\begin{aligned}
& \sum_{j=1}^{M \times N} C_{i j} a_{1 j}=\frac{\partial W}{\partial y}, \quad(i=1,2, \cdots, M \times N) \\
& \sum_{j=1}^{M \times N} C_{i j} a_{2 j}=W, \quad(i=1,2, \cdots, M \times N)
\end{aligned}
$$

$\gamma_{j}$ in Equation (14) is dimensionless vortex strength, then the expression of vortex strength $\gamma_{c}$ is:

$$
\gamma_{c}=a V \gamma_{j}=a V\left(a_{1 j} T(t)+a_{2 j} \frac{T^{\prime}(t)}{V}\right),(j=1,2, \cdots, M \times N)
$$

\section{Analytical Derivation of Additional Mass}

It is assumed that the air is an incompressible and in viscid ideal fluid, and the motion of the air fluid is caused by the membrane vibration [12]. Therefore, the kinetic energy increment of any part of the fluid is equal to the work done by the surface pressure $P$ in $\mathrm{d} t$ time [8].

$$
\mathrm{d} E_{t}=-\iint_{S} V_{N} p \mathrm{~d} S \mathrm{~d} t
$$

where, $E_{t}$ is the kinetic energy, $p$ is the aerodynamic pressure on the membrane surface, $V_{N}$ is the normal velocity of the air particle, which is equal to the normal vibration velocity of the membrane, and the integral region is $S \in\{0 \leq x \leq a, 0 \leq y \leq b\}$, then

$$
\mathrm{d} E_{t}=-\iint_{S} p \frac{\partial w(x, y, t)}{\partial t} \mathrm{~d} x \mathrm{~d} y \cdot \mathrm{d} t
$$

According to reference [11], the kinetic energy change rate of the additional air mass can be expressed as

$$
\frac{\mathrm{d} E_{t}}{\mathrm{~d} t}=m_{a} \iint_{S} \frac{\partial w(x, y, t)}{\partial t} \frac{\partial^{2} w(x, y, t)}{\partial t^{2}} \mathrm{~d} S
$$


where $m_{a}$ is the additional air mass of the membrane $\left(\mathrm{kg} / \mathrm{m}^{2}\right)$, substituting Equations (20) into (21), we can get:

$$
m_{a}=\frac{-\int_{0}^{b} \int_{0}^{a} p \frac{\partial w(x, y, t)}{\partial t} \mathrm{~d} x \mathrm{~d} y}{\int_{0}^{b} \int_{0}^{a} \frac{\partial w(x, y, t)}{\partial t} \cdot \frac{\partial w^{2}(x, y, t)}{\partial t^{2}} \mathrm{~d} x \mathrm{~d} y}
$$

\section{Parameter Analysis and Discussion}

Let the displacement function satisfy the boundary conditions as [14] [15] [16] [17] [18].

$$
w(x, y, t)=a_{0} \sin \omega t \cdot \sin \frac{m \pi x}{a} \sin \frac{n \pi y}{b}
$$

By substituting the displacement function (23) into the aerodynamic expression (8) of the open membrane, the aerodynamic pressure acting on the membrane surface can be obtained as follows:

$$
\begin{aligned}
p= & \rho_{0} \frac{\partial}{\partial t} \int_{0}^{y} a V\left(a_{1 j} a_{0} \sin \omega t+a_{2 j} \frac{\omega a_{0} \cos \omega t}{V}\right) \mathrm{d} \eta \\
& +\rho_{0} V^{2} a\left(a_{1 j} a_{0} \sin \omega t+a_{2 j} \frac{\omega a_{0} \cos \omega t}{V}\right) \\
= & \rho_{0} a V a_{0} \omega \cos \omega t \int_{0}^{y} a_{1 j} \mathrm{~d} \eta-a a_{0} \omega^{2} \sin \omega t \int_{0}^{y} a_{2 j} \mathrm{~d} \eta \\
& +\rho_{0} V^{2} a a_{1 j} a_{0} \sin \omega t+\rho_{0} V a a_{2 j} \omega a_{0} \cos \omega t
\end{aligned}
$$

By substituting Equation (24) with Equation (22), the additional air mass expression of the membrane can be obtained

$$
\begin{aligned}
& m_{a}=\frac{\int_{0}^{b} \int_{0}^{a}\left[\rho_{0} a V a_{0} \omega \cos \omega t \cdot \int_{0}^{y} a_{1 j} \mathrm{~d} \eta-a a_{0} \omega^{2} \sin \omega t \int_{0}^{y} a_{2 j} \mathrm{~d} \eta+\rho_{0} a a_{0} a_{1 j} V^{2} \sin \omega t+\rho_{0} V a a_{0} a_{2 j} \omega \cos \omega t\right] \cdot W \mathrm{~d} x \mathrm{~d} y}{\int_{0}^{b} \int_{0}^{a} W \cdot a_{0} \omega^{2} \sin \omega t \cdot W \mathrm{~d} x \mathrm{~d} y} \\
&= \frac{4 \rho_{0}}{b \omega^{2}} \beta_{1} \cdot V^{2}+\frac{4 \rho_{0}}{b \omega} \cdot \frac{\cos \omega t}{\sin \omega t} \cdot\left(\beta_{2}+\beta_{3}\right) \cdot V-\frac{4}{b} \beta_{4} \\
& \text { where, }
\end{aligned}
$$

$$
\begin{gathered}
\beta_{1}=\int_{0}^{b} \int_{0}^{a} a_{1 j} \sin \frac{m \pi x}{a} \sin \frac{n \pi y}{b} \mathrm{~d} x \mathrm{~d} y \\
\beta_{2}=\int_{0}^{b} \int_{0}^{a} a_{2 j} \sin \frac{m \pi x}{a} \sin \frac{n \pi y}{b} \mathrm{~d} x \mathrm{~d} y \\
\beta_{3}=\int_{0}^{b} \int_{0}^{a} \int_{0}^{y} a_{1 j} \sin \frac{m \pi x}{a} \sin \frac{n \pi y}{b} \mathrm{~d} \eta \mathrm{d} x \mathrm{~d} y \\
\beta_{4}=\int_{0}^{b} \int_{0}^{a} \int_{0}^{y} a_{2 j} \sin \frac{m \pi x}{a} \sin \frac{n \pi y}{b} \mathrm{~d} \eta \mathrm{d} x \mathrm{~d} y
\end{gathered}
$$

\subsection{Static Air Field $(V=0)$}

When the wind speed $V=0$, the variation relationship between the additional air mass and the vibration mode of the open membrane under different cross wind direction span ratio is shown in Figure 5. 
It can be seen from Figure 5 that with the increase of mode number, the additional mass decreases gradually, and the influence of mode on the additional air mass decreases gradually. The reason is that the vibration amplitude of high-order mode of membrane is small, and the influence of aerodynamic force on membrane vibration is relatively weak. The motion of the first-order mode of membrane is volume expansion state, and the vibration amplitude is the largest In addition, the additional mass increases with the increase of the span ratio of the membrane under the same mode.

\subsection{Flowing Air Field $(V \neq 0)$}

Assuming that the wind speed is along the $X$ direction, let $\lambda=b / a$ is the span ratio of the transverse $(Y)$ and longitudinal $(X)$ wind directions; $\gamma=N_{0 x} / N_{0 y}$ is the pretension ratio of the longitudinal $(X)$ and transverse $(Y)$ wind directions. Take the parameters of membrane as

$$
a=20 \mathrm{~m}, f=1 \mathrm{~m}, N_{0 x}=2 \mathrm{kN} / \mathrm{m}, \quad \gamma=1, \lambda=1 .
$$

The curve of the additional mass ratio with the wind speed under different modes is shown in Figure 6. With the increase of wind speed, the additional mass ratio decreases gradually, because in the process of membrane vibration caused by air, the excitation effect of wind increases, while the composition of membrane self-excited vibration decreases. Therefore, the aeroelastic coupling effect between membrane and air is reduced. Still, it can be seen that the first mode has the strongest additional aerodynamic force between the membrane and the surrounding air, which is much larger than the second and the third modes.

It can be seen from Figure 7 that with the increase of pretension, the additional air mass of the membrane decreases gradually, but the reduction amplitude is weaker than that of wind speed, because the increase of pretension makes the vibration stiffness of the membrane increase, so that the vibration response of the membrane becomes smaller under the same wind speed, thus reducing the interaction between the wind and the membrane. On the other hand, it also reflects that when the membrane stiffness is lower, the influence of the additional mass on the vibration process of the membrane cannot be ignored.

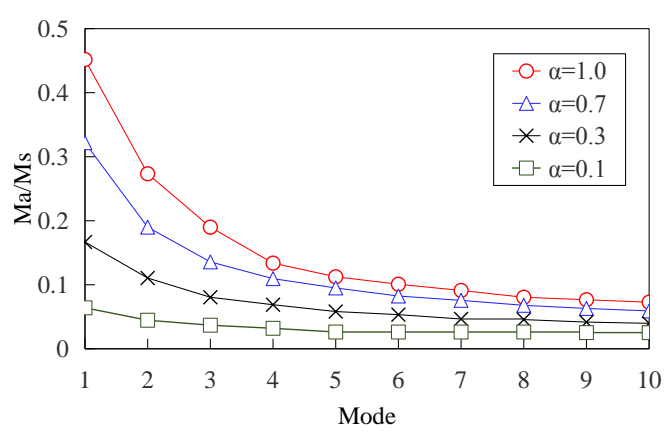

Figure 5. Additional air mass for open membrane. 


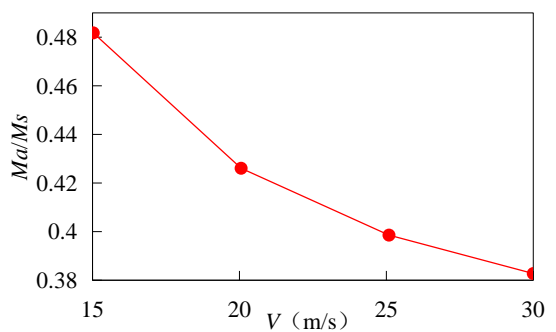

First mode

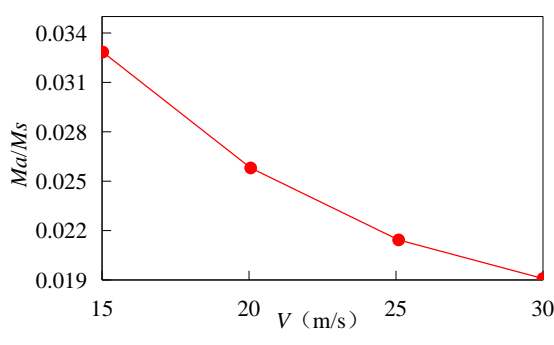

Third mode

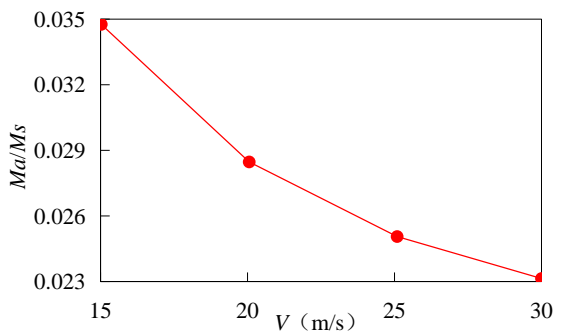

Second mode

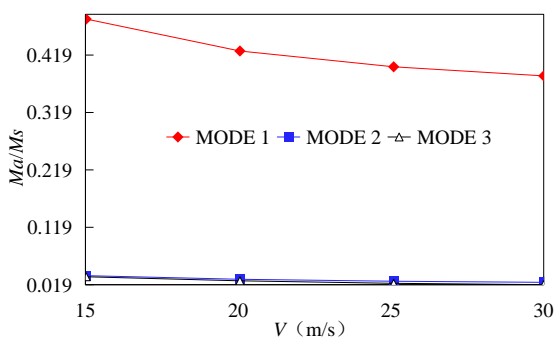

The additional mass ratio

Figure 6. Influence to additional mass of flat membrane with wind speed.

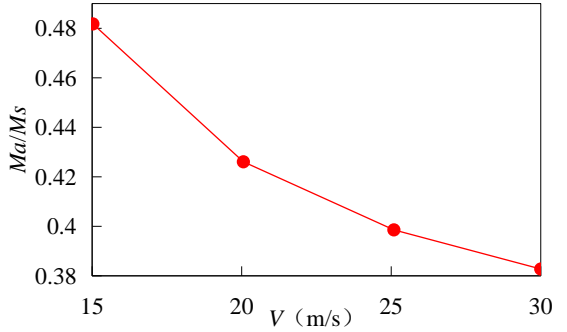

$N_{0 \mathrm{x}}=2 \mathrm{kN} / \mathrm{m}$

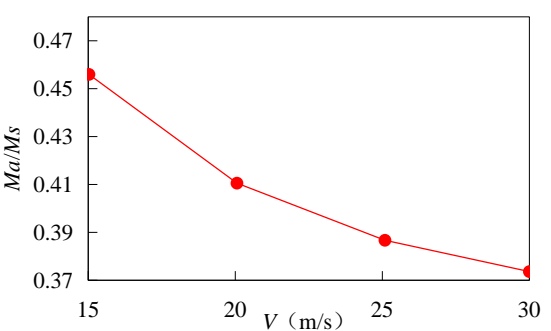

$N_{0 \mathrm{x}}=3 \mathrm{kN} / \mathrm{m}$

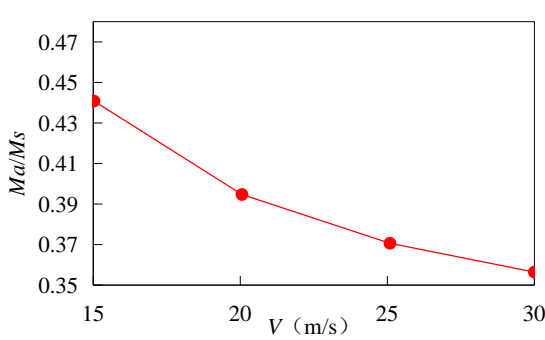

$N_{0 \mathrm{x}}=4 \mathrm{kN} / \mathrm{m}$

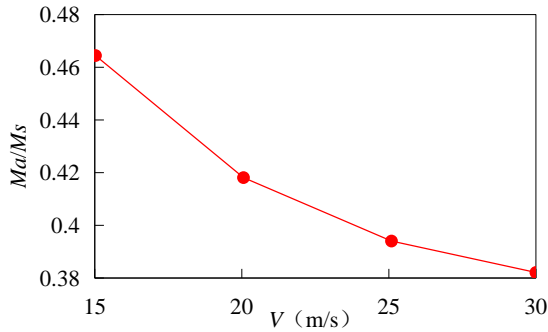

$N_{0 \mathrm{x}}=2.5 \mathrm{kN} / \mathrm{m}$

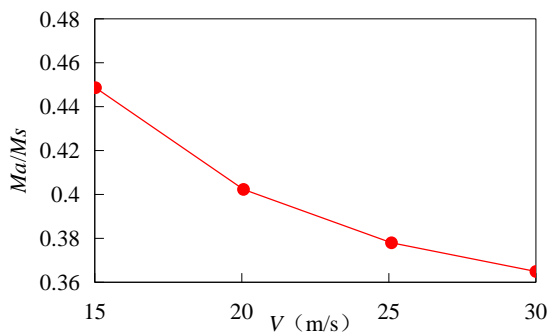

$N_{0 \mathrm{x}}=3.5 \mathrm{kN} / \mathrm{m}$

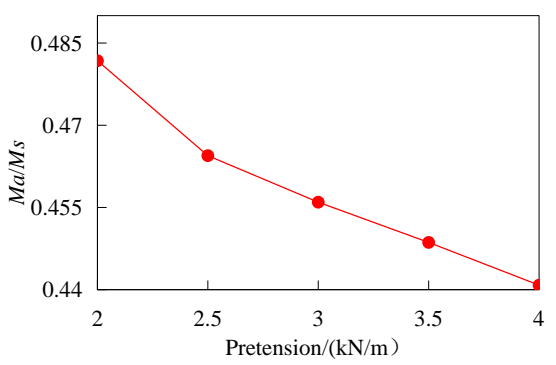

$V=15 \mathrm{~m} / \mathrm{s}$

Figure 7. Influence to additional mass of flat membrane with the initial pretension. 


\section{Conclusions}

According to the first law of thermodynamics and the principle of aeroelasticity, the additional mass of the membrane is solved analytically in this paper, and the parameters are analyzed. It provides a theoretical basis for further study of the aerodynamic damping of membranes and the influence of additional aerodynamic force on the vibration characteristics of membranes. The main conclusions are as follows:

1) The additional mass of the first mode of membrane is the largest, while that of the other three modes is relatively small.

2) With the increase of mode number, the additional mass decreases gradually.

3) In the same mode, the additional mass increases with the increase of the membrane span ratio.

4) The additional mass of the membrane decreases with the increase of pretension, and the decrease amplitude of the high-order mode is smaller than that of the low-order mode.

\section{Acknowledgements}

The work is supported by the National Natural Science Foundation of China (Grant No. 51608060) and the Innovation fund of Hebei University of Engineering (Grant No. SJ010002159).

\section{Data Availability}

The data used to support the findings of this study are available from the corresponding author upon request.

\section{Conflicts of Interest}

The authors declare no conflicts of interest regarding the publication of this paper.

\section{References}

[1] Miyake, A., Yoshimura, T. and Makin, M. (1992) Aerodynamic Instability of Suspended Roof Models. Journal of Wind Engineering and Industrial Aerodynamics, 42, 1471-1482. https://doi.org/10.1016/0167-6105(92)90154-3

[2] Li, Y.Q., Wang, L., Tamura, Y. and Shen, Z.Y. (2009) Wind Tunnel Test on Levy Type Cable Dome. Proceeding of the 7 th Asia-Pacific Conference on Wind Engineering, Taipei, 1-7.

[3] Wang, L., Li, Y.Q. and Shen, Z.Y. (2011) Experimental Investigation on the Added Mass of Membranes Vibrating in Air. Journal of Vibration Engineering, 24, 125-132.

[4] Zhou, Y., Li, Y.Q., Shen, Z.Y., Wang, L. and Tamura, Y. (2014) Numerical Analysis of Added Mass for Open Flat Membrane Vibrating in Still Air Using the Boundary Element Method. Journal of Wind Engineering and Industrial Aerodynamics, 131, 100-111. https://doi.org/10.1016/j.jweia.2014.05.007

[5] Kim, J.Y., Yu, E., Kim, Y.D. and Tamura, Y. (2011) Long-Term Monitoring of 
Wind-Induced Response of a Large-Span Roof Structure. Journal of Wind Engineering and Industrial Aerodynamics, 99, 955-963. https://doi.org/10.1016/j.jweia.2011.06.008

[6] Wu, Y., Sun, X.Y. and Shen, S.Z. (2008) Computation of Wind-Structure Interaction on Tension Structures. Journal of Wind Engineering \& Industrial Aerodynamics, 96, 2019-2032. https://doi.org/10.1016/j.jweia.2008.02.043

[7] Yang, Q.S., Wang, S.J. and Zhu, W.L. (2008) Experimental Study on the Static Interaction between Membrane Structures and Air. China Civil Engineering Journal, 41, 19-25.

[8] Sun, X.Y., Wu, Y. and Shen, S.Z. (2005) Study on Additional Mass and Aerodynamic Damping of Membrane Structure. Proceedings of the 12 th National Academic Conference on Structural Wind Engineering, Xi'an, 46-51.

[9] Li, Q.X. and Sun, B.N. (2007) Influence Regularity of Aerodynamic Forces on Dynamic Characteristics of Membrane Structures. Acta Aerodynamica Sinica, 25, 137-144.

[10] Mao, G.D., Sun, B.N. and Lou, W.J. (2004) Added Air-Mass of Membrane Structures. Engineering Mechanics, 21, 153-158.

[11] Minami, H. (1998) Added Mass of a Membrane Vibrating at Finite Amplitude. Journal of Fluids and Structures, 12, 919-932. https://doi.org/10.1006/jfls.1998.0175

[12] Solari, G. (2019) Wind and Aerodynamics. In: Wind Science and Engineering, Springer International Publishing, Berlin, 441-558.

https://doi.org/10.1007/978-3-030-18815-3 7

[13] Xu, Y.P., Zheng, Z.L., Liu, C.J. and Song, W.J. (2011) Aerodynamic Stability Analysis of Geometrically Nonlinear Orthotropic Membrane Structure with Hyperbolic Paraboloid. Journal of Engineering Mechanics, 137, 759-768.

https://doi.org/10.1061/(ASCE)EM.1943-7889.0000278

[14] Song, W.J., Xu, J., Wang, X.W. and Liu, C.J. (2020) Effect of Geometric Nonlinearity on Membrane Roofs Stability in Air Flow. Shock and Vibration, 2020, Article ID: 2305145. https://doi.org/10.1155/2020/2305145

[15] Zheng, Z.L. and Song, W.J. (2012) Study on Dynamic Response of Rectangular Orthotropic Membranes under Impact Loading. Journal of Adhesion Science and Technology, 26, 1467-1479. https://doi.org/10.1163/156856111X618335

[16] Zheng, Z.L., Xu, Y.P., Liu, C.J., He, X.T. and Song, W.J. (2011) Nonlinear Aerodynamic Stability Analysis of Orthotropic Membrane Structures with Large Amplitude. Structural Engineering and Mechanics, 37, 401-413. https://doi.org/10.12989/sem.2011.37.4.401

[17] He, X.T. and Chen, S.L. (2003) Perturbation Solution to Large Deflection Problem of Cantilever Beams. Journal of Chongqing Jianzhu University, 25, 46-51.

[18] Mohyud-Din, S.T. and Noor, M.A. (2007) Homotopy Perturbation Method for Solving Fourth-Order Boundary Value Problems. Mathematical Problems in Engineering, 2007, Article ID: 98602. https://doi.org/10.1155/2007/98602 


\section{Nomenclatures}

a: length of the membrane

$b$ : width of the membrane

$E_{t}$ : the kinetic energy

$M_{s}$ : areal density of membrane

$M_{a}$ : the additional air mass of the membrane

$N_{0 x}$ : the initial stress of membrane in $X$ direction

$N_{0 y}$ : the initial stress of membrane in $Y$ direction

$P$ : aerodynamic pressure on the membrane surface

$P_{1}$ : the indoor air pressure of the lower surface of the membrane

$P_{2}$ : the outdoor air pressure of the upper surface of the membrane

$T(t)$ : a function of time in the process of vibration

$W(x, y)$ : a function of mode shape

$V:$ wind velocity

$V_{x i}, V_{y i}, V_{z i}$ the velocity components of the upper and lower surfaces

$\phi_{i}$ : the velocity potential function of the upper and lower surface

$\gamma=N_{0 x} / N_{0 y}$ : the pretension ratio of the longitudinal $(X)$ and transverse $(Y)$ wind directions

$\gamma_{c}(x, y, t):$ the vortex density on the surface element

$\gamma_{w}(x, y, t):$ the wake vortex force

$\lambda=b / a$ : the span ratio of the transverse $(Y)$ and longitudinal $(X)$ wind directions;

$\rho_{0}$ : air density. 